\title{
Radiation-Induced Heterogeneous Polymerization. II. Polymerization of Acrylonitrile in the Presence of DMF
}

\author{
Takeshi WAdA and Masaaki TAKeHISA \\ Japan Atomic Energy Research Institute, Takasaki \\ Radiation Chemistry Research Establishment, \\ Takasaki, Gunma, Japan. \\ (Received April 9, 1973)
}

\begin{abstract}
The radiation-induced heterogeneous polymerization of acrylonitrile in the presence of $\mathrm{DMF}$ at $0-80^{\circ} \mathrm{C}$ was studied kinetically. The polymerization proceeded with precipitation of the polymer at mole fractions of the monomer higher than 0.4 . The rate of polymerization in the steady state was proportional to the square root of the dose rate in the presence of DMF in contrast with a dose rate exponent of 0.9 in bulk polymerization. This fact indicates that the first-order termination by radical occlusion does not take place in this heterogeneous polymerization in the same way as in the homogeneous polymerization. This may be due to the larger mobility of the propagating chain due to the presence of DMF. The rate constant of the elementary reactions in the heterogeneous system $(m=0.54)$ at $35^{\circ} \mathrm{C}$ were determined to be $k_{\mathrm{p}}=2.6$, $k_{\mathrm{t}}=4.5 \times 10^{2}$, and $k_{\mathrm{trs}}=1.5 \times 10^{-3}$.

KEY WORDS Heterogeneous Polymerization / Radiation Polymerization / Kinetics / Acrylonitrile / Solution Polymerization / Rate Constant /
\end{abstract}

The bulk polymerization of monomers which act as precipitants for their polymers and the polymerizations of the usual monomers, such as styrene, MMA, and etc., in an excess amount of poor solvents for their polymers are known to proceed by heterogeneous polymerization. In such heterogeneous polymerizations, some noteworthy phenomena different from those in the usual homogeneous polymerization are observed: for example, acceleration in an earlier stage and dose rate (or initiator concentration) exponents of the polymerization rate larger than the 0.5 of typical homogeneous systems. These phenomena have also been observed in the radiation-induced heterogeneous polymerization of acrylonitrile ${ }^{1-5}$ and have been explained by the existence of a long-lived radical and by a first-order termination reaction due to the radical occlusion.

On the other hand, there have been several papers on the radiation-induced homogeneous polymerization of acrylonitrile in the presence of an excess amount of a good solvent $(N, N-$ dimethylformamide or dimethyl sulfoxide). ${ }^{6-8}$
However, no study on the heterogeneous polymerization in the presence of a smaller amount of a good solvent has been reported. This heterogeneous polymerization is interesting as the polymerization in the intermediate state between the bulk polymerization and the homogeneous polymerization.

In this paper, the effects of the mole fraction of monomer and the reaction temperature on the amount of polymerized monomer and on the molecular weight of the polymer, the kinetics of the polymerizations in a heterogeneous and a homogeneous system, and the post polymerization were studied. The results are compared with those of bulk polymerization reported previously. ${ }^{5}$

\section{EXPERIMENTAL}

Acrylonitrile and $N, N$-dimethylformamide (DMF) were used for the polymerization, after distillation of commercial reagents from Kanto Chemical Co. Inc. These reagents were pipetted in a glass ampoule of $10-\mathrm{mm}$ diameter, degassed 


\section{T. WADA and M. TAKEHISA}

5 times by repeated freezing-pumping-thawing cycles using a vacuum line, and sealed off under vacuum.

To carry out the polymerization the ampoules were immersed in a temperature-controlled bath and were irradiated by a cobalt-60 $\gamma$-radiation source. After the irradiation, the polymerization was stopped by placing the ampoules in liquid nitrogen. The reaction mixture was poured into an excess amount of acetone and then the polymer precipitated was separated from the monomer and the solvents with a glass filter, washed well with acetone on the glass filter, dried at $60^{\circ} \mathrm{C}$ under vacuum for $24 \mathrm{hr}$, and weighed.

Within $5 \mathrm{~min}$ of halting the irradiation at $35^{\circ} \mathrm{C}$ the post-polymerization was carried out in a temperature controlled bath at $80^{\circ}$ or $35^{\circ} \mathrm{C}$ by standing for a required period.

The molecular weight of the polymer was determined from the intrinsic viscosity in DMF at $35^{\circ} \mathrm{C}$ by use of following equation:

$$
[\eta]=2.10 \times 10^{-3} \bar{M}_{n}^{0.625}
$$

\section{RESULTS}

Figure 1 shows the effect of the mole fraction of the monomer $(m)$ on the polymerized monomer and the molecular weight of the polymer in the presence of DMF. The polymerization proceeded with precipitation of the polymer at mole fractions of the monomer higher than about 0.4. The polymer yield in the homogeneous system gradually increased with the increase in the mole fraction of monomer. The yield for the heterogeneous system in DMF rapidly increased with increasing mole fraction of monomer from 0.5 to 0.95 and then decreased in bulk. The molecular weight of the polymer increased smoothly with the increase in the monomer mole fraction.

Figure 2 shows the Arrhenius plots of the conversion and the molecular weight of the polymer in the heterogeneous polymerization for a monomer mole fraction of 0.54 . These plots indicate a break point at about $30^{\circ} \mathrm{C}$. From the results of the polymer yield, the apparent activation energy of polymerization is $6.6 \mathrm{kcal} / \mathrm{mol}$ $\left(0-30^{\circ} \mathrm{C}\right)$ and $2.1 \mathrm{kcal} / \mathrm{mol}\left(30-60^{\circ} \mathrm{C}\right)$. The molecular weight of the polymer shows a maximum at about $30^{\circ} \mathrm{C}$.

The results of post polymerization of acryl-

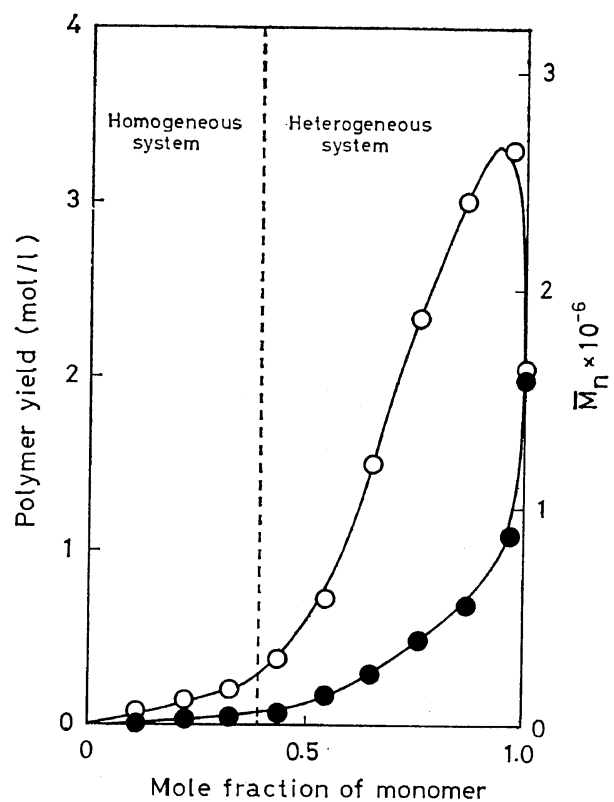

Figure 1. The polymer yield $(O)$ and the molecular weight (O) at various mole fractions of monomer. Reaction conditions: temperature, $35^{\circ} \mathrm{C}$, dose rate, $2.9 \times 10^{4} \mathrm{rad} / \mathrm{hr}$; and time $1.0 \mathrm{hr}$.

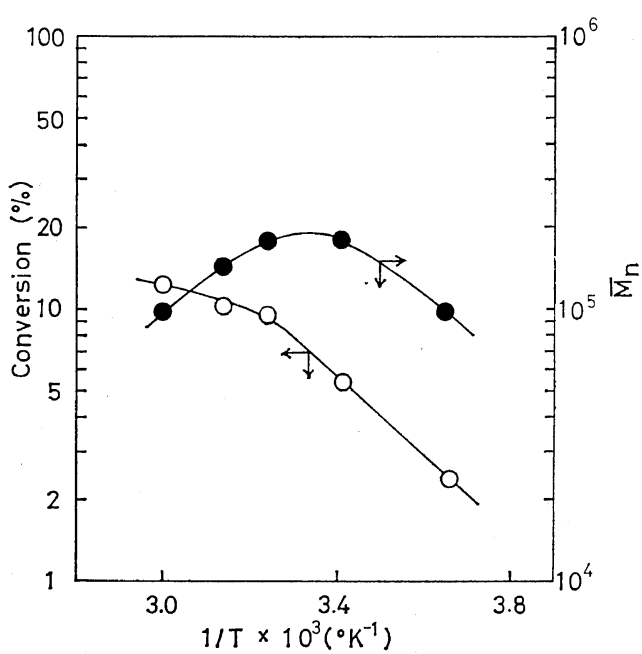

Figure 2. Arrhenius plot for heterogeneous polymerization of acrylonitrile in DMF. Reaction conditions and symbols are the same as in Figure 1.

onitrile in two different heterogeneous systems (the bulk polymerization and the polymerization in monomer mole fraction of 0.54 ) are summarized in Table I. When the reaction mixture was 
Radiation-Induced Heterogeneous Polymerization

Table I. Post-polymerization of AN in Bulk and in DMFa

\begin{tabular}{|c|c|c|c|c|c|c|}
\hline \multirow{2}{*}{$\mathrm{AN}, \mathrm{m} l$} & \multirow{2}{*}{$\mathrm{DMF}, \mathrm{m} l$} & \multicolumn{2}{|c|}{ Post-polym. } & \multirow{2}{*}{ Polymer, g } & \multirow{2}{*}{ Conv, $\%$} & \multirow{2}{*}{$\bar{M}_{n} \times 10^{-4}$} \\
\hline & & Temp, ${ }^{\circ} \mathrm{C}$ & Time, $\min$ & & & \\
\hline 10 & 0 & - & - & 0.965 & 11.8 & 110 \\
\hline 10 & 0 & 35 & 60 & 1.053 & 12.9 & 78 \\
\hline 10 & 0 & 80 & 1 & 1.067 & 13.1 & 121 \\
\hline 10 & 0 & 80 & 5 & 1.548 & 19.0 & - \\
\hline 10 & 0 & 80 & 22 & 1.414 & 17.4 & 49 \\
\hline 10 & 0 & 80 & 60 & 1.601 & 19.6 & 36 \\
\hline 10 & 10 & - & - & 0.778 & 9.6 & 18.0 \\
\hline 10 & 10 & 80 & 22 & 0.783 & 9.6 & 16.4 \\
\hline 10 & 10 & 80 & 60 & 0.775 & 9.5 & 15.4 \\
\hline
\end{tabular}

a In-source polymerization conditions: dose rate, $2.9 \times 10^{4} \mathrm{rad} / \mathrm{hr}$; temp, $35^{\circ} \mathrm{C}$; time, $1.0 \mathrm{hr}$.

heated up to $80^{\circ} \mathrm{C}$ after the pre-irradiation at $35^{\circ} \mathrm{C}$ for $1 \mathrm{hr}$, the post polymerization took place in the bulk system, but not at all in the heterogeneous system with DMF. The post polymerization in bulk proceeded quickly at $80^{\circ} \mathrm{C}$ and slowly at $35^{\circ} \mathrm{C}$.

Figures 3, 4, 5, and 6 show the polymer yield and the molecular weight of the polymer against reaction time in the heterogeneous system ( $m=$ 0.54 ) and in the homogeneous system ( $m=0.22)$.

In the heterogeneous polymerizations, the acceleration period was shorter than that in bulk

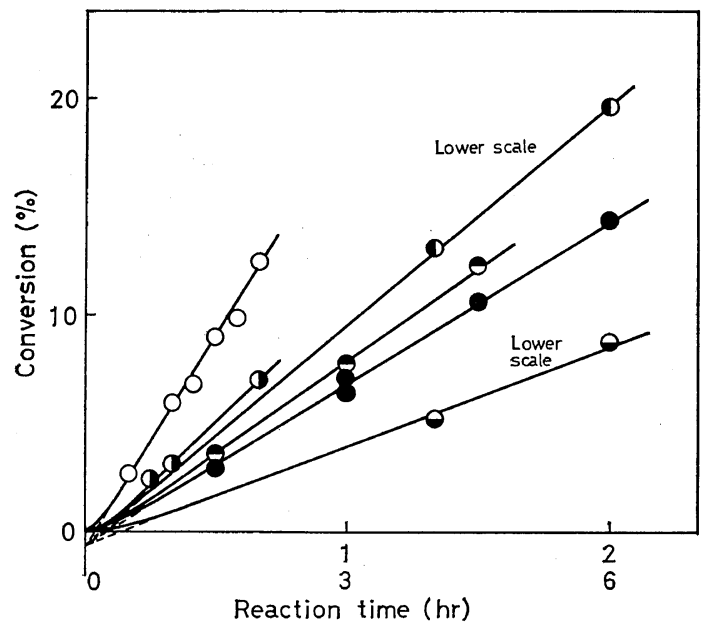

Figure 3. Time-conversion curves of heterogeneous polymerization of acrylonitrile in DMF. Reaction conditions: monomer mole fraction, 0.54 ; temperature, $35^{\circ} \mathrm{C}$; time, $2.0 \mathrm{hr}$; dose rate $\times 10^{-4}$

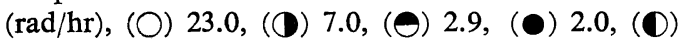
0.68 , (९) 0.29 . reported previously. $^{5}$ After the acceleration period in the early stage the polymerization proceeded steadily; all the intercepts on the ordinate of the extrapolated lines of the linear portions in the steady state were constant, independent of the dose rate. The molecular weight of the polymer increased gradually in the early stage and then became almost constant.

In the homogeneous system $(m=0.22)$, the polymerization proceeded linearly from the initial stage and the molecular weight of polymer was constant, regardless of the conversion.

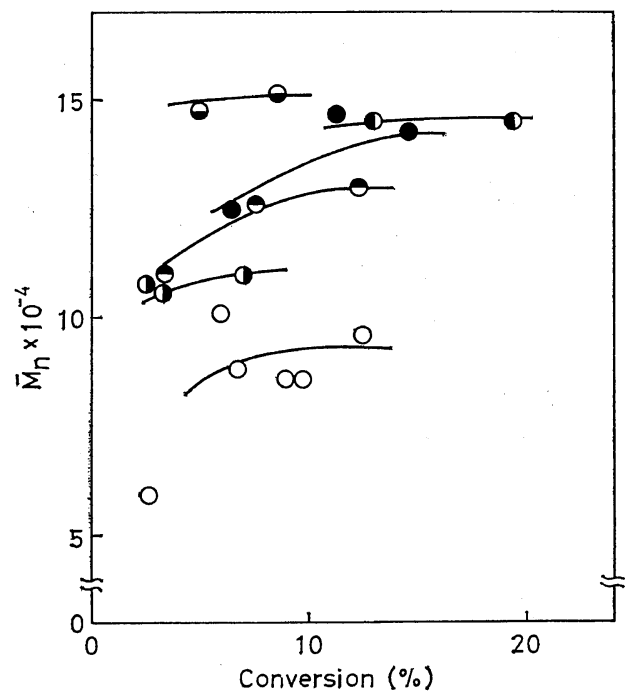

Figure 4. Number-average molecular weight of the polymer $v s$. conversion in heterogeneous polymerization of acrylonitrile in DMF. Reaction conditions and symbols are the same as in Figure 3. 


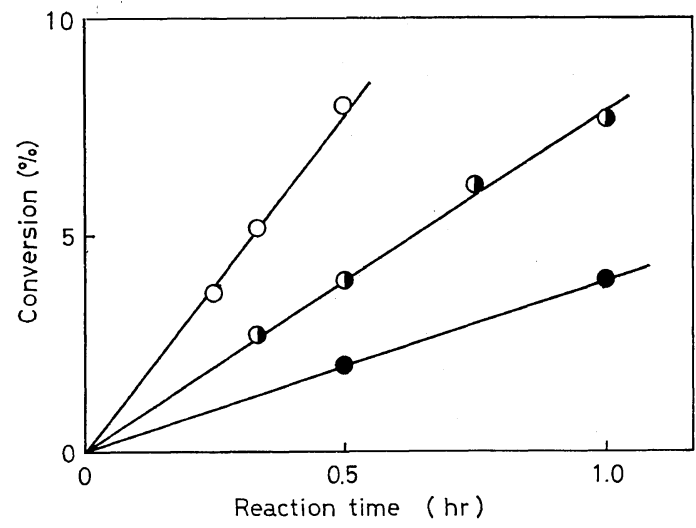

Figure 5. Time-conversion curves of homogeneous polymerization of acrylonitrile in DMF. Reaction conditions: monomer mole fraction 0.22 ; temp, $35^{\circ} \mathrm{C}$; dose rate $\times 10^{-4},(\mathrm{rad} / \mathrm{hr}),(\bigcirc) 23.0$, (๑) 7.0 , (॰) 2.0 .

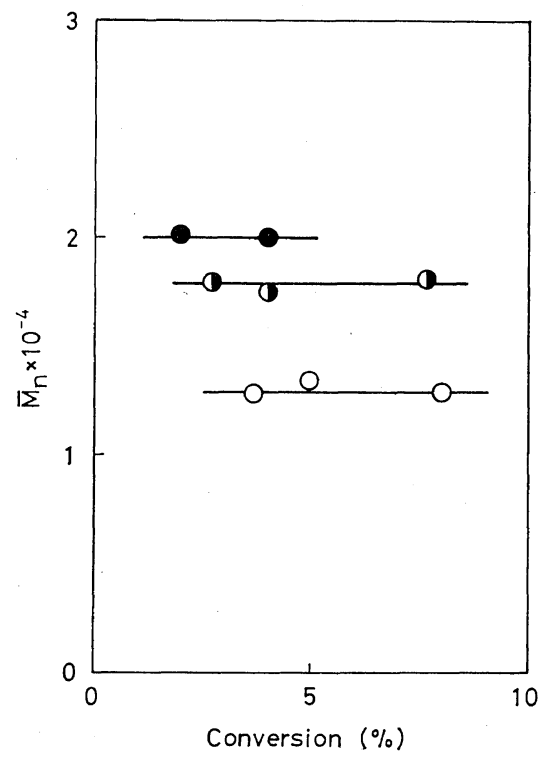

Figure 6. Number-average molecular weight of the polymer vs. conversion, in homogeneous polymerization of acrylonitrile in DMF. Reaction conditions and symbols are the same as in Figure 5.

\section{DISCUSSION}

As shown in Figure 7, the rate of polymerization in the steady state was proportional to the square root of the dose rate in the homogeneous and also the heterogeneous systems $(m=0.22$ and $0.54)$. This suggests that the first-order termination

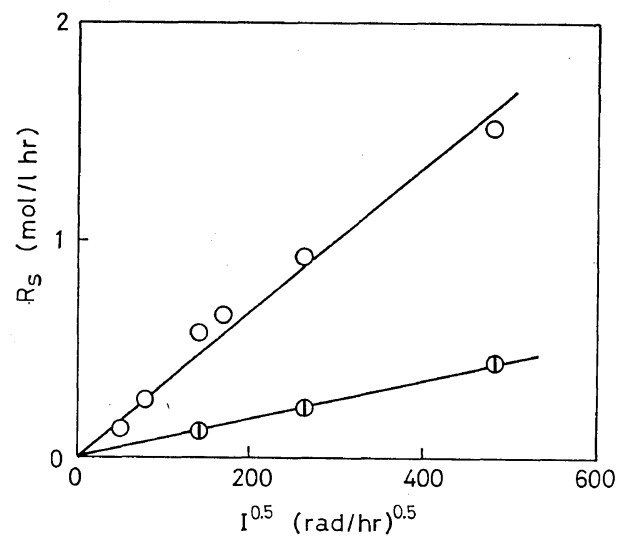

Figure 7. The rate of polymerization at steady state $v s$. square root of dose rate. Symbols: (O) heterogeneous polymerization $(m=0.54)$, (1) homogeneous polymerization $(m=0.22)$.

by radical occlusion does not take place in the heterogeneous polymerization in the presence of DMF in the same way as in the homogeneous polymerization. Furthermore, the experimental results of the post polymerization leads to the same conclusion. The post polymerization in bulk is thought to take place by some long-lived radical such as the occluded one, as was reported by Bamford, et al. ${ }^{9}$ Therefore, the absence of the occluded radical in the heterogeneous polymerization with DMF is also supported by the fact that the post polymerization does not take place in this system.

The elementary reaction can be written as follows;

Initiation

$$
\begin{aligned}
& \mathrm{M} m \mathrm{R}^{\cdot} \\
& \mathrm{S} m \mathrm{R}^{\cdot} \\
& R_{\mathrm{i}}=\left(k_{\mathrm{im}}[\mathrm{M}]+k_{\mathrm{is}}[\mathrm{S}]\right) I
\end{aligned}
$$

Propagation

$$
\begin{aligned}
& \mathbf{R}_{n}^{\cdot}+\mathbf{M} \rightarrow \mathbf{R}_{n+1}^{\cdot} \\
& R_{\mathrm{p}}=k_{\mathrm{p}}\left[\mathbf{R}^{\cdot}\right][\mathrm{M}]
\end{aligned}
$$

Chair-transfer

$$
\begin{aligned}
& \mathbf{R}_{n}^{\cdot}+\mathbf{M} \rightarrow \mathrm{P}_{n}+\mathbf{R}_{i} \\
& \mathbf{R}_{n}+\mathbf{S} \rightarrow \mathbf{P}_{n}+\mathbf{R}_{i} \\
& R_{\mathrm{tr}}=\left(k_{\mathrm{trm}}[\mathrm{M}]+k_{\mathrm{trs}}[\mathrm{S}]\right)\left[\mathbf{R}^{\cdot}\right]
\end{aligned}
$$

Termination

$$
\begin{aligned}
& \mathbf{R}_{m}+\mathbf{R}_{n}^{\cdot} \rightarrow \mathbf{P}_{m+n} \\
& \boldsymbol{R}_{\mathrm{t}}=k_{\mathrm{t}}\left[\mathbf{R}^{\cdot}\right]^{2}
\end{aligned}
$$


Here $\mathrm{M}$ and $\mathrm{S}$ are monomer and solvent, respectively; $\mathbf{R}_{n}^{\cdot}$ is a propagating radical composed of $n$-monomers and $\mathrm{P}_{n}$ is a polymer composed of $n$-monomers; $R_{\mathrm{i}}, R_{\mathrm{p}}, R_{\mathrm{tr}}$, and $R_{\mathrm{t}}$ are the rates of initiation, propagation, chain transfer, and termination; $k_{\mathrm{im}}, k_{\mathrm{is}}, k_{\mathrm{p}}, k_{\mathrm{trm}}, k_{\mathrm{trs}}$, and $k_{\mathrm{t}}$ are the rate constants.

On the basis of the mechanism described above, the amount of polymerized monomer at the steady state $M_{\mathrm{p}}$ is expressed by

$$
M_{\mathrm{p}}=R_{\mathrm{s}} t-b
$$

where

$$
\begin{gathered}
R_{\mathrm{s}}=\frac{k_{\mathrm{p}}[\mathrm{M}]\left(k_{\mathrm{im}}[\mathrm{M}]+k_{\mathrm{is}}[\mathrm{S}]\right)^{0.5} I^{0.5}}{k_{\mathrm{t}}^{0.5}}=a I^{0.5} \\
b=\frac{2 k_{\mathrm{p}}[\mathrm{M}] \ln 2}{k_{\mathrm{t}}}
\end{gathered}
$$

As shown in equation 14, the value of $b$ is a constant independent of the dose rate. The result of the heterogeneous polymerization in Figure 3 is consistent with the relationship of eq 12 ; it gives $b=0.061 \mathrm{~mol} / l$. The value of $b$ in homogeneous polymerization is very small, because of $k_{\mathrm{t}} \gg k_{\mathrm{p}}[\mathrm{M}]$, and cannot be obtained. The slope of the straight line shown in the plot of $R_{\mathrm{s}}$ against $I^{0.5}$ (Figure 7) gives the value of $a$ based on eq 13. The values in the homogeneous and in the heterogeneous polymerizations are obtained as $9.2 \times 10^{-4}$ and $3.5 \times 10^{-3} \mathrm{~mol} / \mathrm{l} \mathrm{hr}{ }^{0.5} \mathrm{rad}^{0.5}$.

On the other hand, the relationship for the number average of the degree of polymerization is expressed by

$$
1 / \overline{D P}=c R_{\mathrm{s}}+d
$$

where

$$
\begin{gathered}
c=\frac{k_{\mathrm{t}}}{2 k_{\mathrm{p}}^{2}[\mathrm{M}]^{2}} \\
d=\frac{k_{\mathrm{trm}}[\mathrm{M}]+k_{\mathrm{trs}}[\mathrm{S}]}{k_{\mathrm{p}}[\mathrm{M}]}
\end{gathered}
$$

Figures 8 and 9 show the plot of $1 / \overrightarrow{D P}$ against $R_{\mathrm{S}}$ at various dose rates in homogeneous and heterogeneous systems, respectively. From these figures, $c=1.65 \times 10^{-4} \mathrm{hr} l / \mathrm{mol}$ and $d=3.25 \times 10^{-4}$ in the heterogeneous system, and $c=4.4 \times 10^{-3} \mathrm{hr} l / \mathrm{mol}$ and $d=2.1 \times 10^{-3}$ in the homogeneous system.

Rate constants of each elementary reaction in the heterogeneous system $(m=0.54)$ were determined with the values of $a, b, c$, and $d$ as follows: $k_{\text {im }}[\mathrm{M}]+k_{\text {is }}[\mathrm{S}]=4.0 \times 10^{-9} \mathrm{~mole} / l \mathrm{rad}, \quad k_{\mathrm{p}}=2.6 l /$ mol sec, $k_{\mathrm{t}}=4.5 \times 10^{2} l / \mathrm{mole} \mathrm{sec}$, and $k_{\mathrm{trm}}[\mathrm{M}]+$

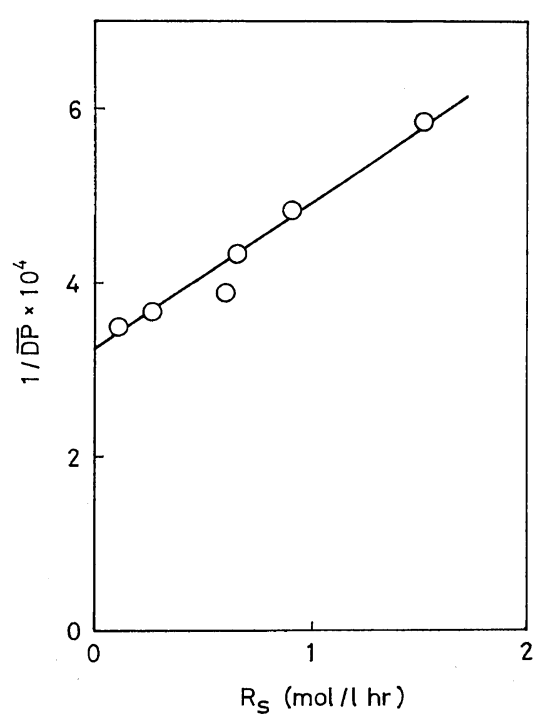

Figure 8. $1 / \overline{D P}$ vs. $R_{\mathrm{S}}$ in heterogeneous polymerization of acrylonitrile $(m=0.54)$.

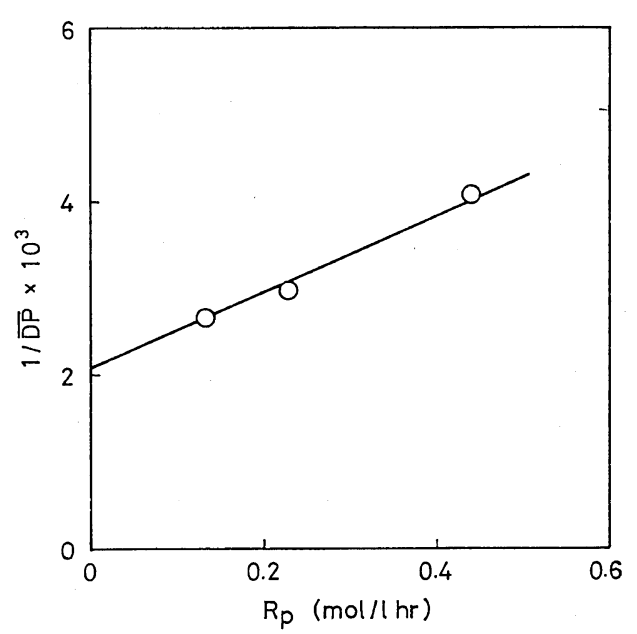

Figure 9. $1 / \overline{D P} v s . R_{\mathrm{S}}$ in homogeneous polymerization of acrylonitrile $(m=0.22)$.

$k_{\mathrm{trs}}[\mathrm{S}]=6.3 \times 10^{-3} \mathrm{sec}^{-1}$. The value of the rate constant of initiation by DMF was obtained as $k_{\text {is }}=5.9 \times 10^{-10} \mathrm{rad}^{-1}$ based on the value of $k_{\mathrm{im}}[\mathrm{M}]+k_{\mathrm{is}}[\mathrm{S}]$ in this experiment and $k_{\mathrm{im}}=$ $2.9 \times 10^{-11} \mathrm{rad}^{-1}$ in bulk polymerization as reported in the previous paper. From the value of $k_{\text {is }}$, the $G$-value of initiation for DMF is calculated as 7.8 , which is about 15 times the $G$-value for acrylonitrile $(0.53) .{ }^{5}$ The value of $k_{\mathrm{p}}$ is similar to that in the bulk and the value 


\section{T. WADA and M. TAKehisA}

of $k_{\mathrm{t}}$ is about twice that in the bulk. These results are thought to show that the swelling of a propagating polymer chain by DMF considerably affects the termination by recombination, while it affects the propagation only slightly. If the relation $k_{\mathrm{trs}}[\mathrm{S}] \gg k_{\mathrm{trm}}[\mathrm{M}]$ holds, $k_{\mathrm{trs}}=1.5 \times$ $10^{-3} l /$ mole sec is obtained.

On the other hand, for the homogeneous polymerization $(m=0.22) k_{\text {im }}[\mathrm{M}]+k_{\text {is }}[\mathrm{S}]=7.4 \times 10^{-9}$ $\mathrm{mol} / l \mathrm{rad}, k_{\mathrm{p}}[\mathrm{M}] / k_{\mathrm{t}}{ }^{0.5}=0.059(\mathrm{~mol} / l \mathrm{sec})^{0.5}$, and $\left(k_{\mathrm{trm}}[\mathrm{M}]+k_{\mathrm{trs}}[\mathrm{S}]\right) / k_{\mathrm{p}}=6.3 \times 10^{-3} \mathrm{~mol} / l$. In this polymerization $k_{\mathrm{is}}=7.1 \times 10^{-10} \mathrm{rad}^{-1}$ is obtained based on $k_{\mathrm{im}}=2.9 \times 10^{-11} \mathrm{rad}^{-1}$ in the bulk. This value is close to the value of $k_{\text {is }}$ in the heterogeneous polymerization $(m=0.54)$. Colebourne et $a l .^{7}$ determined $k_{\mathrm{p}}$ and $k_{\mathrm{t}}$ at 0,25 and $66^{\circ} \mathrm{C}$ in the radiation-induced homogeneous polymerization in DMF $(m=0.10)$, using the rotatingsector technique. From these values $k_{\mathrm{p}}$ and $k_{\mathrm{t}}$ at $35^{\circ} \mathrm{C}$ are estimated, respectively, as about $4.5 \times 10^{2}$ and $5.0 \times 10^{7}$. The value of $k_{\mathrm{p}} / k_{\mathrm{t}}{ }^{0.5}$ (0.064) calculated on the basis of these values of $k_{\mathrm{p}}$ and $k_{\mathrm{t}}$ shows close agreement with the value obtained in this polymerization. This seems to indicate that the values of $k_{\mathrm{p}}$ and $k_{\mathrm{t}}$ in the present homogeneous system are almost equal to the values obtained by Colebourne, et al. ${ }^{7}$ Figure 10 shows the value of $1 / \overline{D P}-k_{\mathrm{t}} R_{\mathrm{s}} / 2 k_{\mathrm{p}}{ }^{2}[\mathrm{M}]^{2}$ against $[\mathrm{S}] /[\mathrm{M}]$ at various monomer mole fractions in the homogeneous system. This plot gives the values of the chain transfer constants on the basis of

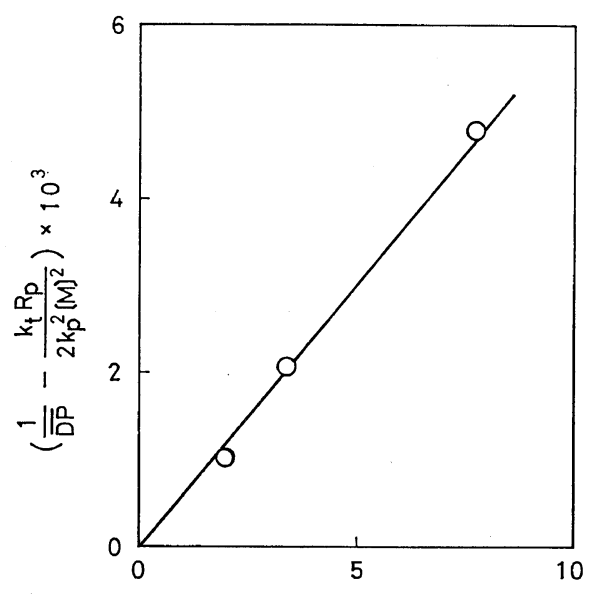

$[\mathrm{S}] /[\mathrm{M}]$

Figure 10. $\left[(1 / \overline{D P})-\left(k_{\mathrm{t}} R_{\mathrm{s}} / 2 k_{\mathrm{p}}{ }^{2}[\mathrm{M}]^{2}\right)\right] v s .[\mathrm{S}] /[\mathrm{M}]$ in homogeneous polymerization. eq 18.

$$
\frac{1}{\overline{D P}}-\frac{k_{\mathrm{t}} R_{\mathrm{s}}}{2 k_{\mathrm{p}}^{2}[\mathrm{M}]^{2}}=C_{\mathrm{m}}+C_{\mathrm{s}} \frac{[\mathrm{S}]}{[\mathrm{M}]}
$$

As shown in Figure 10, the plot gives a straight line crossing the origin. This shows that the value of $C_{\mathrm{m}}$ is very small. From the slope of the straight line the value of $C_{\mathrm{s}}$ was obtained as $6.0 \times 10^{-4}$, which coincided with the value of $C$ $\left(6.1 \times 10^{-4}\right)$ obtained by the value of $d$ based on the assumption $k_{\mathrm{trs}}[\mathrm{S}] \gg k_{\mathrm{trm}}[\mathrm{M}]$. If the value of $k_{\mathrm{p}}$ is $4.5 \times 10^{2}$, as estimated from the results of Colebourne, $k_{\mathrm{trs}}=0.27 \mathrm{lmole} / \mathrm{sec}$ is found. In several papers, ${ }^{9-13}$ the values of $C_{\mathrm{s}}$ for DMF in homogeneous system have been determined at $50-60^{\circ} \mathrm{C}$. These values are in the range of $10^{-4}$ to $10^{-3}$, which agree with the value of $C_{\mathrm{s}}$ in this homogeneous polymerization. Most of values of $C_{\mathrm{m}}$ at $25-60^{\circ} \mathrm{C}$ in homogeneous systems ${ }^{14-17}$ are reported to be $1-3 \times 10^{-5}$, which are significantly smaller than the value of $C_{\mathrm{s}}$ for DMF.

Consequently, it became clear that each rate constant (except for $k_{\mathrm{i}}$ ) of an elementary reaction in the heterogeneous system is very much smaller than that in the homogeneous one. The ratio of $k_{\mathrm{t}}$ in the homogeneous and heterogeneous systems is about $10^{5}$ and is larger than the ratio of $k_{\mathrm{p}}$ or $k_{\text {trs }}$ (about 200). These results lead to the conclusion that the physical state of the propagating chain, whether it is precipitated or dissolved, strongly affects the values of the rate constant. Similar results were obtained for the polymerization of MMA in homogeneous and heterogeneous systems by Hayden, et al..$^{18}$ However the transfer constants in both systems are almost equal to one another. Significant differences between the heterogeneous system in bulk and that in DMF ( $m=$ 0.54 ) were not observed for the rate constants of $k_{\mathrm{p}}$ and $k_{\mathrm{t}}$, but the first-order termination by the occlusion of propagating radical observed in bulk does not take place in the presence of DMF.

The effect of monomer mole fraction on the results of polymerization shown in Figure 1 is considered as follows. The change of polymer yield in homogeneous polymerization is mainly attributed to the change of monomer concentration. However, the change of polymer yield with monomer mole fraction in the heterogeneous system is thought to be attributed to the change of rate constants with the change of physical 
state of the propagating chain, in addition to the change of monomer concentration. The rapid increase in the polymer yield with increasing monomer mole fraction from 0.4 to 0.95 is explained by the larger decrease in the value of $k_{\mathrm{t}}$ relative to that in $k_{\mathrm{p}}$. Furthermore, the decrease in the polymer yield in bulk is attributed to the presence of the first-order termination by radical occlusion.

Since the rate of chain-transfer is considerably larger than that of termination in the presence of DMF, the molecular weight of the polymer is determined mainly by the ratio of the rate of propagation and that of chain transfer. In addition, as described above, the transfer constant in the heterogeneous and homogeneous systems is almost equal. Therefore, the increasing molecular weight of the polymer with the increase in monomer mole fraction can be attributed to the increasing ratio of the monomer to the solvent.

As shown in Figure 2, the effects of temperature on the yield and on the molecular weight of the polymer in the heterogeneous system $(m=$ $0.54)$ are interesting. In the bulk polymerization, also, it is known that the Arrhenius plot shows a break point similar to the present experiment. In radiation-induced polymerization, Bernas, et $a l .{ }^{4}$ have reported that the apparent activation energy is $13.5 \mathrm{kcal} / \mathrm{mole}$ at temperatures lower than $60^{\circ} \mathrm{C}$ and $1.7 \mathrm{kcal} / \mathrm{mole}$ in the temperature range of 70 to $90^{\circ} \mathrm{C}$, respectively, and that the molecular weight is highest at about $60^{\circ} \mathrm{C}$. On the other hand, the activation energies in homogeneous systems were reported as $6.3 \mathrm{kcal} / \mathrm{mole}$ $\left(-38-20^{\circ} \mathrm{C}\right)$ for radiation-induced polymerization $^{8}$ and as about $5 \mathrm{kcal} / \mathrm{mole} \cdot\left(9-41^{\circ} \mathrm{C}\right)$ for the photopolymerization. ${ }^{19}$ These apparent activation energies for radiation- and photo-induced polymerizations of acrylonitrile under various conditions are summarized in Table II, together with the results of the present experiment.

As the activation energy for initiation is nearly zero in radiation- and photo-induced polymerization, the apparent activation energy for the polymerization is determined mainly by the activation energies of propagation and termination, that is, $E_{\mathrm{p}}-\frac{1}{2} E_{\mathrm{t}}$. The activation energy at temperatures lower than $30^{\circ} \mathrm{C}$ in the present experiment agrees with that in the homogeneous case, while that at temperatures higher than $30^{\circ} \mathrm{C}$
Table II. The apparent activation energies for radiation- and photo-induced polymerization of acrylonitrile

\begin{tabular}{|c|c|c|}
\hline Reaction system & $\begin{array}{l}\text { Monomer } \\
\text { mole } \\
\text { fraction }\end{array}$ & $\begin{array}{l}\text { Apparent activation } \\
\text { energy, } \mathrm{kcal} / \mathrm{mol}\end{array}$ \\
\hline $\begin{array}{l}\text { Homogeneous } \\
\text { polymerization } \\
\text { in DMF }\end{array}$ & $\begin{array}{l}0.33 \\
0.10\end{array}$ & $\begin{array}{l}6.3\left(-38 \sim 20^{\circ} \mathrm{C}\right)^{8} \\
5.0\left(9 \sim 41^{\circ} \mathrm{C}\right)^{19}\end{array}$ \\
\hline $\begin{array}{l}\text { Heterogeneous } \\
\text { polymerization } \\
\text { in bulk }\end{array}$ & 1.0 & $\begin{array}{r}13.5\left(20 \sim 60^{\circ} \mathrm{C}\right)^{4} \\
1.7\left(70 \sim 90^{\circ} \mathrm{C}\right)^{4}\end{array}$ \\
\hline $\begin{array}{l}\text { Heterogeneous } \\
\text { polymerization } \\
\text { in DMF }\end{array}$ & 0.54 & $\begin{array}{l}6.6\left(0 \sim 30^{\circ} \mathrm{C}\right) \\
2.1\left(30 \sim 60^{\circ} \mathrm{C}\right)\end{array}$ \\
\hline
\end{tabular}

in the present experiment agrees with that at temperatures higher than $70^{\circ} \mathrm{C}$ in the bulk.

These tendencies can be explained by the following consideration. It seems that the mobility of the propagating chain radical at temperatures lower than $30^{\circ} \mathrm{C}$ is depressed and, therefore, little affected by temperature. Accordingly, the activation energy for termination at temperatures lower than $30^{\circ} \mathrm{C}$ is thought to be negligible. On the other hand, a small activation energy for termination is known for homogeneous polymerization, in which the propagating chain is perfectly free. Consequently, the similar apparent activation energies at temperatures lower than $30^{\circ} \mathrm{C}$ in the heterogeneous polymerization with DMF and in the homogeneous polymerization would be attributed mainly to the propagation step. The higher apparent activation energy at temperatures lower than $60^{\circ} \mathrm{C}$ in the bulk can be explained by the existence of the first-order termination by radical occlusion. On the other hand, the lower activation energy at temperatures higher than $30^{\circ} \mathrm{C}$ in the heterogeneous system with DMF and at temperatures higher than $60^{\circ} \mathrm{C}$ in bulk are thought to be based on the increase of both propagation and termination rates with increasing temperature. The diffusion process may affect the termination because of the swelling effect on the propagating chain at higher temperatures and, therefore, the effect of temperature on the termination reaction is larger than that at lower temperatures. The lower break point of the Arrhenius plot in the present experiment, compared to that in bulk polymerization, will be due 
to the increased mobility of the propagating chain, because of the presence of DMF.

Some remarkable differences between the heterogeneous polymerization in the presence of DMF and the homogeneous one in an excess amount of DMF or the heterogeneous one in bulk have been elucidated mainly as differences of mobility of the propagating chain.

\section{REFERENCES}

1. Berstein, E. C. Farmer, W. C. Rothchild, and F. F. Spalding, J. Chem. Phys., 21, 1303 (1953).

2. A. Chapiro and J. Sebban-Dannon, J. Chim. Phys., 54, 776 (1957).

3. A. Prevot-Bernas and J. Sebban-Dannon, ibid., 53, 418 (1957).

4. H. Bernas and M. Bodard, J. Polym. Sci., 48, 167 (1960).

5. T. Wada, T. Watanabe, and M. Takehisa, Polymer J,. 4, 136 (1973).

6. Bensasson and Prevot-Bernas, J. Chim. Phys., 53, 93 (1956).

7. N. Colebourne, E. Collinson, D. J. Currie, and
F. S. Dainton, Trans. Faraday Soc., 59, 1357 (1963).

8. I. S. Bhardwaj, A. Chapiro, and L. Perec, Eur. Polym. J., 7, 135 (1971).

9. C. H. Bamford, A. D. Jenkins, R. Johnstone, and E. F. T. White, Trans. Faraday Soc., 55, 168 (1959).

10. J. T. Clarke, R. O. Howard, and W. H. Stockmayer, Makromol. Chem., 44/46, 427 (1961).

11. H. Kikuchi and M. Watanabe, Kobunshi Kagaku (Chem. High Polymers), 21, 37 (1964).

12. N. T. Srinvasan and M. Santnappa, Makromol. Chem., 26, 80 (1958).

13. W. M. Thomas, E. H. Gleason, and J. J. Pellon, J. Polym. Sci., 17, 275 (1955).

14. P. F. Onyon, ibid., 22, 19 (1956).

15. J. Ulbricht, Z. Phys. Chem, 221, 346 (1962).

16. C. H. Bamford, A. D. Jenkins, and F. F. T. White, J. Polym. Sci., 34, 271 (1959).

17. S. K. Das, S. R. Chatterjee, and S. R. Palit, Proc. Royal Soc., Ser. A (London), 227, 252 (1955).

18. P. Hayden and H. Melville, J. Polym. Sci., 43, 215 (1960).

19. P. F. Onyon, Trans. Faraday Soc., 52, 80 (1956). 